\title{
Partial Hearing Preservation after Translabyrinthine Vestibular Schwannoma Resection: Case Report and Review of the Literature
}

\author{
Syed F. Ahsan ${ }^{1}$ Dennis Bojrab Jr. ${ }^{2}$ Robert Standring ${ }^{1}$ \\ ${ }^{1}$ Department of Otolaryngology, Henry Ford Health System, Detroit, \\ Michigan, United States \\ 2 Department of Otolaryngology, Wayne State University, Detroit, \\ Michigan, United States \\ Address for correspondence Syed F. Ahsan, MD, Department of \\ Otolaryngology, Henry Ford Hospital, 2799 W. Grand Blvd, Detroit, MI \\ 48202, United States (e-mail: sahsan1@hfhs.org).
}

J Neurol Surg Rep 2015;76:e211-e215.

\begin{abstract}
Objectives To describe a unique case report of a patient who had partial hearing preservation after translabyrinthine (TL) removal of a vestibular schwannoma (VS).

Study Design Case report.

Methods The patient's chart was reviewed for hearing levels before and after surgery. Preoperative magnetic resonance imaging (MRI) was compared with postoperative MRI for determination of completeness of tumor removal. The literature on hearing preservation after TL resection is reviewed.

Keywords

- translabyrinthine vestibular schwannoma resection

- vestibule

- hearing preservation

- translabyrinthine resection

Results A 42-year-old woman underwent a TL removal of a VS. The patient's preoperative pure tone average (PTA) was $70 \mathrm{~dB}$ and word recognition score (WRS) was $40 \%$. Postoperatively, the patient was able to hear ambient noise in the surgical ear. Her bone conduction PTA was $70 \mathrm{~dB}$, but the WRS score dropped to $2 \%$. One year later, she continues to hear ambient noise and sound in the operative ear.

Discussion This is the fifth reported case of partial hearing preservation after TL VS resection. It suggests that by preserving the vestibule and the fluids within the vestibule when possible, there maybe enough residual auditory neural structures for a traditional cochlear implant to benefit such a patient. In addition, preserving the incus when possible may help maintain air conduction to help patients with sound localization.
\end{abstract}

\section{Introduction}

The translabyrinthine (TL) approach for resection of a vestibular schwannoma (VS) is considered the most reliable method for complete tumor removal. In addition, it is generally associated with better facial nerve function. ${ }^{1,2}$ However, it is thought to lead to complete hearing loss. The other two common approaches, middle cranial fossa (MCF) and retrosigmoid (RS), are more difficult with a worse angle of dissection and with greater risk to the facial nerve due to its location during the procedure (MCF) and a greater risk of incomplete tumor removal in the lateral internal auditory canal (IAC) for the retrosigmoid approach. These two approaches are considered hearing sparing with better hearing preservation reported in the MCF approach for small $<1 \mathrm{~cm}$ tumors that do not extend to the fundus. With larger tumors, the RS approach is advocated when hearing preservation is a possibility. However, in medium to large tumors (Hanover stage T3 $[2.0-3.5 \mathrm{~cm}]$ and $\mathrm{T} 4[>3.5 \mathrm{~cm}]$ ), the hearing preservation rate using the RS approach is $44 \%$ and $18 \%$, respectively. ${ }^{3}$

In 1991, McElveen et al presented the first case of hearing preservation using a modified TL approach. ${ }^{4}$ Their modification involved the attempt to preserve the "pars inferior" received

October 29, 2014

accepted

April 9, 2015

published online

August 4, 2015
DOI http://dx.doi.org/

10.1055/s-0035-1554931. ISSN 2193-6358.
@ 2015 Georg Thieme Verlag KG
Stuttgart · New York

License terms

(®) $\Theta \circledast$ 
e212 Hearing Preservation after Translabyrinthine Resection Ahsan et al.

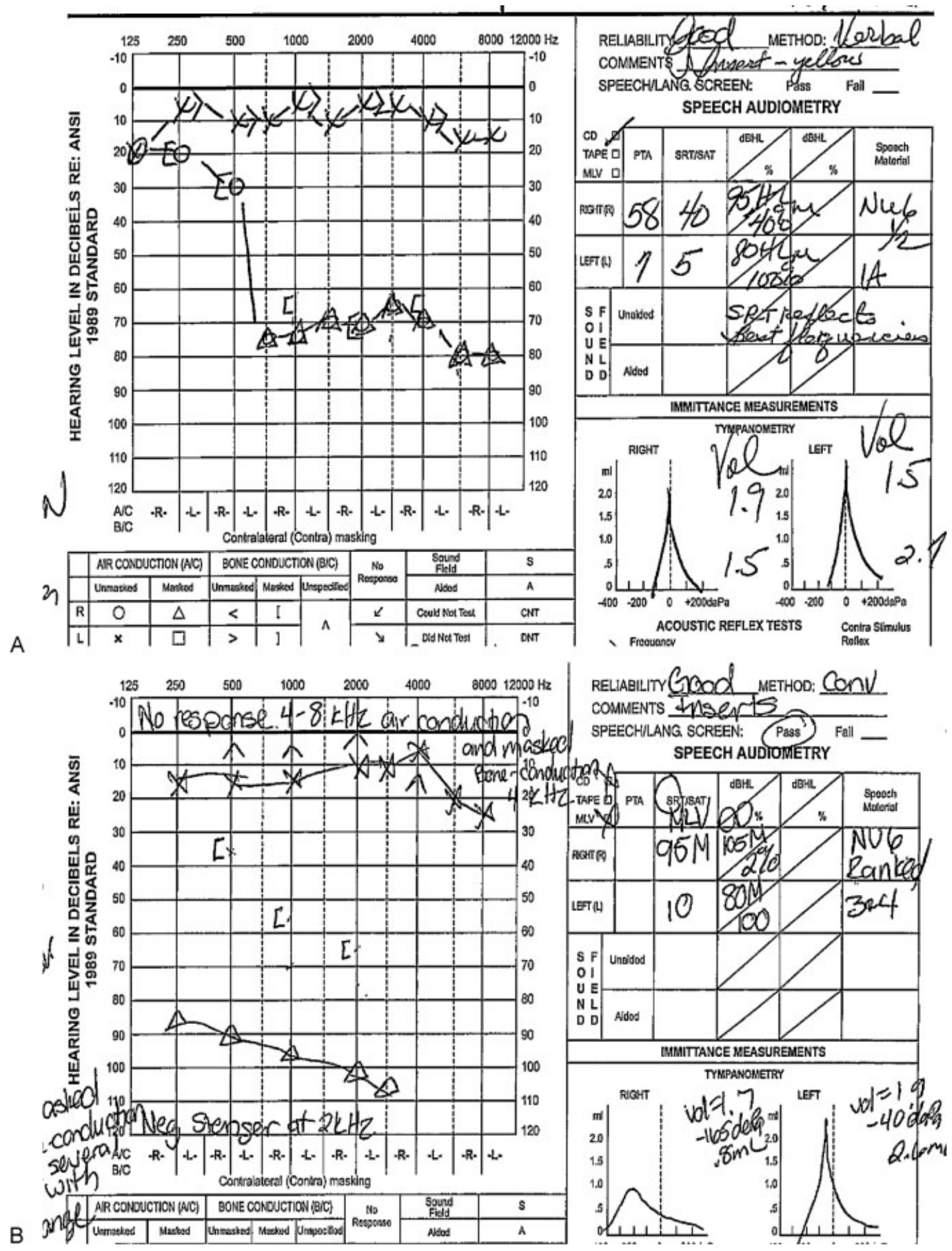

Fig. 1 (A) Preoperative audiogram (right ear: speech reception threshold [SRT]: 40; word recognition score [WRS]: 40\%). (B) Two-month postoperative audiogram (right ear: SRT: 95; WRS: $2 \%$ ).

portion of the inner ear and filling the vestibule with saline. Since then, there have been a few reports of hearing preservation after a classical TL acoustic neuroma resection. ${ }^{5-8}$ To our knowledge, our article presents the fifth case of a patient with partial hearing preservation after a classical TL resection of a VS. In addition, we discuss the implication of partial hearing preservation and the possibility of cochlear implan- tation in selected cases. Institutional review board approval was obtained before proceeding with the article.

\section{Case Report}

A 42-year-old very obese woman presented for evaluation of right-side tinnitus and hearing loss that had been present for 

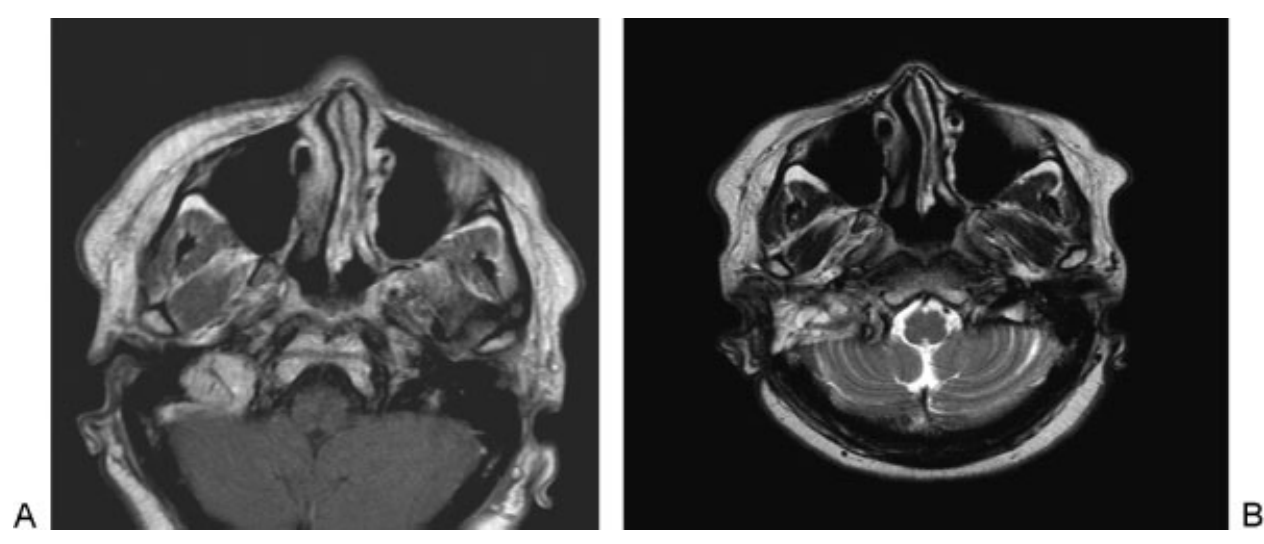

Fig. 2 Magnetic resonance imaging of the brain and internal auditory canal. (A) Before resection. (B) After resection.

6 months. She denied any dizziness or vertigo and had not had similar problems before. Audiogram showed a sloping moderate high-frequency sensorineural hearing loss (SNHL). A subsequent magnetic resonance imaging (MRI) of the brain revealed a right-side cerebellopontine angle (CPA) enhancing mass most likely a VS. It measured $10.6 \times 9.7 \mathrm{~mm}$. The patient was presented with options of observation, stereotactic radiotherapy, and microsurgical resection. She opted for observation and was closely followed. In the following few months, she developed deep venous thrombosis of her lower extremities and was placed on anticoagulation. Unfortunately, during later follow-up, her hearing worsened. A repeat MRI showed growth of the tumor. It increased in size from $10.6 \times 9.7 \mathrm{~mm}$ to $16 \times 11 \mathrm{~mm}$. After her other medical problems were controlled and she was weaned off the anticoagulant, the patient decided to have the VS surgically removed. Her preoperative hearing test showed significant SNHL in the right ear, the speech reception threshold (SRT) was $40 \mathrm{~dB}$, and word recognition score (WRS) was $40 \%$ (-Fig. 1A). Preoperative auditory brainstem response (ABR) was abnormal, and videonystagmogram indicated right-sided $24 \%$ reduced vestibular response. It was recommended due to the size of the tumor, the degree of her hearing loss, and her body habitus that the TL approach was her best option. She obtained a second opinion at another institution, and a similar treatment was recommended. The patient underwent surgery in February 2012 where a classical TL approach was used. Intraoperatively, she had a very high riding jugular bulb that was decompressed, retracted gently with a Freer elevator, and bone wax was placed to keep the bulb depressed and out of the way. The view was still quite narrow due to a very anterior sigmoid sinus. During the surgery, no attempt was made to preserve hearing. However, we packed the area of the vestibule with fat, and Tisseel as well as bone wax was placed over the open vestibule. Moreover, the CPA vasculature was left intact when possible. Eighth nerve monitoring was not used in this case. A subtotal removal of the tumor was performed due to the adherence of the deep part of the tumor capsule to the facial nerve. It was believed that 90\% of the tumor was removed (-Fig. 2). Care was taken in delivering the tumor out of the IAC and preserving the acoustic nerve along with the facial nerve. The incus was removed, the middle ear and eustachian tube was plugged with muscle, fat, and Tisseel, and the antrum was sealed with bone wax.

Postoperative facial nerve function was House-Brackmann (HB) $4 / 6$ that worsened to a HB $5 / 6$ by the week 4 follow-up. Her postoperative imbalance gradually improved. By the week 5 , her facial nerve function started to improve, and the patient stated that she could hear sounds out of her right ear. By postoperative month 3, facial nerve function improved to HB $2 / 6$, and her audiogram at 2 months revealed that she had persistent hearing in the operative ear (-Fig. 1B). The SRT decreased from 40 to $95 \mathrm{~dB}$ due to a significant air-bone gap as a result of the removal of the incus. The bone conduction pure tone average (average of $\mathrm{BC} \mathrm{HL}$ at $500 \mathrm{~Hz}, 1 \mathrm{kHz}$, and 2 $\mathrm{kHz}$ ) was $65 \mathrm{~dB}$, a decrease of $7 \mathrm{~dB}$ from a preoperative value of $58 \mathrm{~dB}$. However, the WRS decreased to $2 \%$ at $105 \mathrm{~dB}$ presentation. For the patient, it was usable in day-to-day routines such as driving and being able to discern the location of sounds. She has subsequently been fitted with a Soundbite device. One year later, her hearing was stable at the lowest frequencies (-Fig. 3). She continues to use the Soundbite device. Her facial function is normal (HB 1/6).

\section{Discussion}

The TL approach is one of the most common methods used in the removal of a VS or a CPA tumor. In counseling patients, it is common to inform them that the chance of hearing preservation is almost $0 \%$. However, there have been a limited number of case reports demonstrating some preservation of hearing after a classical TL approach to acoustic neuroma resection. ${ }^{5-8}$ The present report adds the fifth case to the literature of preservation of some hearing after TL resection. The patients presented by Smith et $\mathrm{al}^{5}$ and Springborg et $\mathrm{al}^{7}$ eventually lost their hearing 12 months and 6 years later, respectively. The patients in the other two reports (Rizvi and Goyal ${ }^{6}$ and Tringali et $\mathrm{al}^{8}$ ) had stable hearing 2 years and 5 years, respectively, after surgery.

McElveen et $\mathrm{al}^{4}$ demonstrated that hearing can be preserved after a modified TL excision. Using intraoperative ABR, 


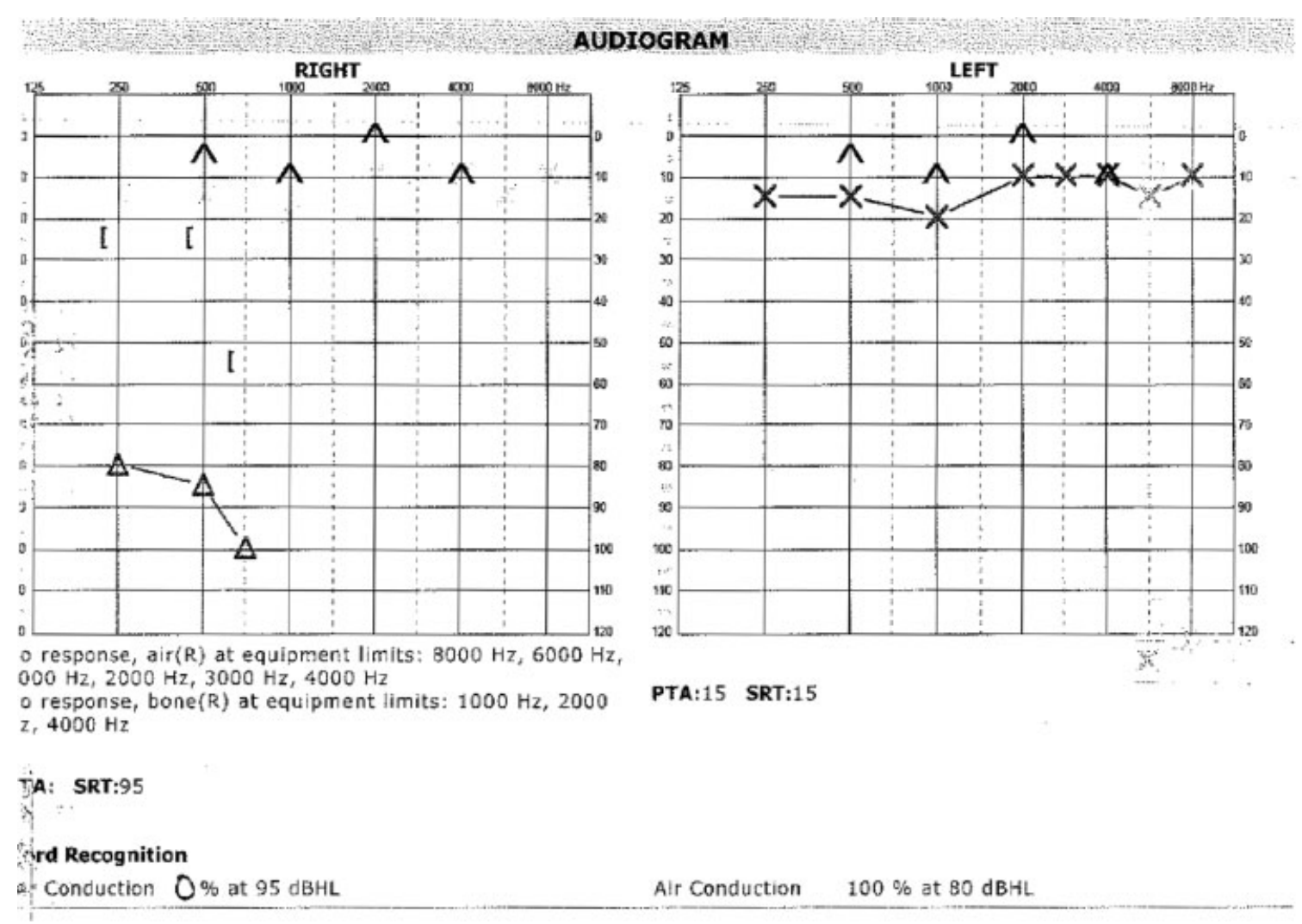

Fig. 3 Postoperative audiogram at 1 year (right ear: speech reception threshold: 95; word recognition score: $0 \%$ ).

they maintained the vasculature and cochlear nerve integrity. They preserved the saccule and cochlea, and used bone wax to seal the vestibule after opening it. Later they filled the vestibule with Ringer lactate and sealed off the labyrinth with bone wax. One of their two patients lost hearing at 1month follow-up. The second patient was successfully treated with diuretics for fluctuating hearing loss.

The common belief is that removal of the labyrinth and opening of the vestibule results in deafness. However, our patient's result as well as that of the previously mentioned reports suggests that, in these patients, deafness is not an absolute certainty. This is in accordance with animal studies demonstrating that hearing can be preserved after ablation of the semicircular canals or partial labyrinthectomy. ${ }^{9-11}$ Several studies have also shown that hearing can be preserved in humans after injury or destruction of the semicircular canals. ${ }^{12-14}$ The challenge is we can routinely preserve some hearing in these patients undergoing "destructive" surgery. Our patient maintained her bone conduction values to an appreciable level with an air-bone gap occurring due to need for incus removal for packing the eustachian tube and middle ear space. However, her speech discrimination decreased significantly. It is however, conceivable that there are preserved spiral ganglion cells. It has been shown that up to $75 \%$ of spiral ganglion cells can be damaged without an increase in pure tone thresholds. ${ }^{15}$ This suggests a mechanism for the success of concurrent cochlear implantation after TL acoustic neuroma resection as first described by Ahsan et al. ${ }^{16}$
The factors that seem to be most important for hearing preservation are preservation of the cochlear nerve, CPA vasculature, maintenance of a fluid-filled vestibule, and preservation of endolymphatic fluids. ${ }^{4,7,17}$ Smith et al suggested that the collapse of the saccule and/or the ductus reuniens may have preserved the cochlear fluids. ${ }^{5}$ Rizvi et al suggested that in their patient, while attempting to seal off the middle ear with bone wax, they may have sealed off the ductus reuniens, which contributed to the stability of the hearing in their patient. ${ }^{6}$ Further study needs to be done to accurately define the critical steps needed in preserving hearing in TL cases.

\section{References}

1 Shelton C, Brackmann DE, House WF, Hitselberger WE. Middle fossa acoustic tumor surgery: results in 106 cases. Laryngoscope 1989;99(4):405-408

2 Arriaga MA, Chen DA. Facial function in hearing preservation acoustic neuroma surgery. Arch Otolaryngol Head Neck Surg 2001;127(5):543-546

3 Samii M, Matthies C. Management of 1000 vestibular schwannomas (acoustic neuromas): hearing function in 1000 tumor resections. Neurosurgery 1997;40(2):248-260; discussion 260-262

4 McElveen JT Jr, Wilkins RH, Erwin AC, Wolford RD. Modifying the translabyrinthine approach to preserve hearing during acoustic tumour surgery. J Laryngol Otol 1991;105(1):34-37

5 Smith PG, Bigelow DC, Kletzker GR, Leonetti JP, Pugh BK, Mishler ET. Hearing preservation following a transtemporal resection of an 
acoustic schwannoma: a case report. Am J Otol 1993;14(5): 434-436

6 Rizvi SS, Goyal RN. Case report and discussion of hearing preservation after translabyrinthine excision of small acoustic tumors. Am J Otol 1999;20(2):249-252

7 Springborg LK, Springborg JB, Thomsen J. Hearing preservation after classical translabyrinthine removal of a vestibular schwannoma: case report and literature review. J Laryngol Otol 2007; 121(1):76-79

8 Tringali S, Ferber-Viart C, Gallégo S, Dubreuil C. Hearing preservation after translabyrinthine approach performed to remove a large vestibular schwannoma. Eur Arch Otorhinolaryngol 2009;266(1): $147-150$

9 Kristensen HK. Acoustic-vestibular and histologic examinations in guinea-pigs after interruption of membranous labyrinth in semicircular canals or cochlea. Acta Otolaryngol 1960; 51:382-402

10 Lempert J, Meltzer PE, Rambo JH, Wever E. The effects of injury to the lateral semicircular canals. Trans Am Acad Ophthalmol Otolaryngol 1956;60(5):718-727
11 Smouha EE, Inouye M. Partial labyrinthectomy with hearing preservation: frequency-specific data using tone-burst auditory brain stem response. Otolaryngol Head Neck Surg 1999;120(2):146-152

12 Canalis RF, Gussen R, Abemayor E, Andrews J. Surgical trauma to the lateral semicircular canal with preservation of hearing. Laryngoscope 1987;97(5):575-581

13 Jahrsdoerfer RA, Johns ME, Cantrell RW. Labyrinthine trauma during ear surgery. Laryngoscope 1978;88(10):1589-1595

14 Bumsted RM, Dolan KD, Sadé J, McCabe BF. Preservation of cochlear function after extensive labyrinthine destruction. Ann Otol Rhinol Laryngol 1977;86(2 pt. 1):131-137

15 Schuknecht HF, Woellner RC. An experimental and clinical study of deafness from lesions of the cochlear nerve. J Laryngol Otol 1955; 69(2):75-97

16 Ahsan S, Telischi F, Hodges A, Balkany T. Cochlear implantation concurrent with translabyrinthine acoustic neuroma resection. Laryngoscope 2003;113(3):472-474

17 Magliulo G, Parrotto D, Stasolla A, Marini M. Modified translabyrinthine approach and hearing preservation. Laryngoscope 2004;114(6):1133-1138 